\title{
A Probabilistic Algorithm for MANET Clustering
}

\author{
Fahimeh Dabaghi-Zarandi ${ }^{1}$, Behrouz Minaei-Bidgoli ${ }^{2}$ and Zohreh Davarzani ${ }^{3}$ \\ ${ }^{1}$ Faculty of Computer Engineering Department, Vali-e-Asr University of Rafsanjan, \\ Rafsanjan, Iran \\ ${ }^{2}$ Faculty of School of Computer Engineering, Iran University of Science \& \\ Technology, Tehran, Iran \\ ${ }^{3}$ Faculty of Computer Engineering Department, Payame Noor University, Tehran, \\ Iran \\ f.dabaghi@vru.ac.ir,b_minaei@iust.ac.ir,davarzani.z@pnurazavi.ac.ir
}

\begin{abstract}
Mobile ad hoc network (MANET) is a type of ad hoc network that MANET nodes can change their locations and configure by themselves on the fly. Because of mobility the MANET nodes, the management of a large MANET is difficult, therefore, clustering in a MANET is an important technique. A large network is divided into several sub networks applying clustering method. When the topology of the network is dynamic and ad hoc, the process of clustering is very complicated. In this paper, we propose a Probabilistic Algorithm for MANET Clustering $(P A M C)$ to improve the performance of this wireless technology. We simulate our algorithm and evaluate it based on two criteria: the average number of clusters and the average reaffiliation.
\end{abstract}

Keywords: Mobile ad hoc network, MANET, Clustering, Re-affiliation

\section{Introduction}

Mobile ad hoc networks are wireless, infrastructure less, multi-hop, dynamic network established by a collection of mobile nodes which provide significant features to the modern communication technologies and services. With the above code of ethics these networks are particularly important and useful in battlefields, military/police exercise, mine site operations and disaster relief operations, emergency search and rescue and so on [1]. Clustering is an important technique in MANET. In this technique, a large network can be divided into several sub-networks with only a few cluster heads maintain the local information [2]. In the cluster formation, every group of nodes near together arranged to be in one group which is used to prevent the flood of unnecessary packets and avoids wasting network bandwidth.

In each cluster, there exist three types of nodes as Cluster Head, Cluster Gateway and Cluster Member. These types are defined as following and an example of clustered network is shown in Figure 1.

Cluster Head (CH): $\mathrm{CH}$ is responsible for cluster management functions and providing hierarchical routing. CHs may be rotated among the members of the cluster to provide load balancing and fault tolerance [3].

Cluster Gateway (CG): CG is a node that connects two clusters. Each gateway may have two CHs. 

[4].

Cluster Member (CM): CM is a node that is neither a cluster head nor a cluster gateway

The aim of clustering algorithm is to elect some appropriate nodes as cluster head then they manage their CMs. Any $\mathrm{CH}$ should collect the cluster information that its neighbors use them in routing process. In routing protocol based on clustering, the route messages are graceful by the following method: Source $\rightarrow$ Cluster Head $\rightarrow$ Gateway $\rightarrow$ Cluster Head $\rightarrow$ Gateway $\rightarrow \ldots$ $\rightarrow$ Destination [5]. [3]:

The main advantages to be gained by clustering an ad hoc wireless network are as follows

1) A virtual backbone consisting of $\mathrm{CHs}$ and the $\mathrm{CG}$ nodes provides an efficient method of routing in ad hoc networks as it forms a simple topology to maintain. This method of hierarchical routing results in smaller routing tables, which are stored by the $\mathrm{CHs}$ and the CG nodes only, and hence efficiency of routing is improved.

2) Messages needed to update routing tables are reduced.

3) Usage of clustering also provides efficient MAC protocols by improving the throughput, scalability, and power consumption.

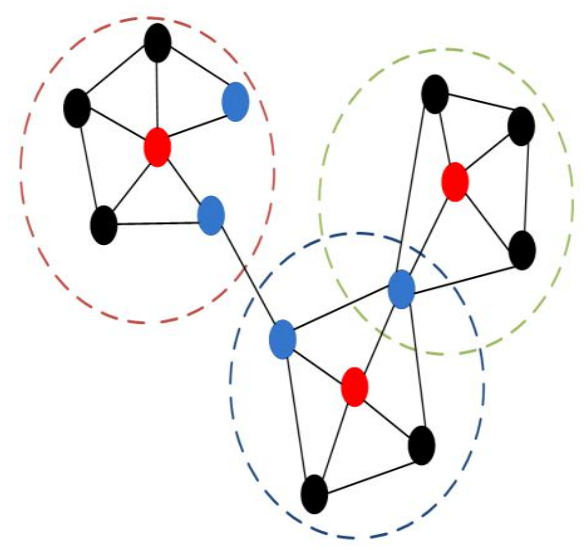

Figure 1. Example of Clustered Network: the Black Nodes are Cluster Member; the Red Nodes are Cluster Head; the Blue Nodes are Cluster Gateway

The reminder of this paper has four sections. Section 2 explains some related clustering algorithms in MANET. Section 3 describes our proposed algorithm. Section 4 explains simulation environment and results and comparing them with the prior clustering algorithms and the conclusion is described in section 5 .

\section{Related Works}

In clustering algorithm, the aim is to elect some nodes as cluster head that this election should be satisfied some constraints such as Average Number of Clusters (ANoC) and average RE-affiliation (RE). In a clustering algorithm, RE should be minimized in optimal case, because a MANET topology is dynamic. ANoC should be minimized with one constraint that the number of nodes in one cluster should not be very increased because the managing all cluster information will be difficult by $\mathrm{CH}$. Selection of optimum number of $\mathrm{CHs}$ is NP-hard, for this reason, node identifier, node degree, node mobility, and its battery power are 
frequently used for the selection of $\mathrm{CHs}$ heuristically [3]. This section contains some clustering algorithms that are explained briefly as following.

Lowest-ID (LID) algorithm is the MANET clustering algorithm that originally was proposed by Baker and Ephremides [6]-[8]. In MANET, each node periodically broadcast some information including its identifier during network, therefore each node receive a message from all neighbors periodically. Each node selects its $\mathrm{CH}$ based on all of messages that has received from neighbors. The neighbor with the lowest identifier is marked by the node as its $\mathrm{CH}$.

Highest-Degree (HD) was originally proposed by Gerla and Parekh [9]-[10]. In this algorithm, a neighbor with the highest degree is marked by the node as its $\mathrm{CH}$. If there exist two neighbors with highest degree, the neighbor with lowest ID is selected as its $\mathrm{CH}$.

Erciyes et al. [11] proposed the clustering algorithm based on spanning tree structure. At first, a spanning tree is constructed based on the network topology then during traversing spanning tree, each node with its children in two next levels are inserted in one cluster.

The Weighted Clustering Algorithm (WCA) [12]-[13] was originally proposed by $\mathrm{M}$. Chatterjee et al. In this algorithm, the election of $\mathrm{CH}$ is based on the weight of each node that is assigned based on several parameters such as degree, distance from neighbors, mobility, and so on.

Other weighted based clustering algorithm was proposed by Mohammad Shayesteh et al. [14]. This algorithm for calculating node weights considers four parameters such as relative speed, stability, number of nodes moving towards a node and battery remaining. Then, CHs are determined based on the weight of nodes.

In [15]-[16] proposed two algorithms that are called distributed clustering algorithm (DCA) and distributed mobility adaptive clustering algorithm (DMAC). In these approaches, each node is assigned weight based on that the node may be a CH or CM. DCA is not applicable in the static networks and not supported in MANET, but DMAC is applicable in MANET.

Selvam et al. [17] proposed a weighted based clustering algorithm. The weight of each node is assigned based on the number of multi-cast neighbors with distance of one hop and two hop.

\section{Proposed Method}

As mentioned in Section 2, the aim of MANET clustering algorithm is to elect some of appropriate nodes as $\mathrm{CH}$. Almost, all of clustering algorithms select $\mathrm{CH}$ s deterministically. In other words, at first, one criterion is selected then the election of $\mathrm{CH}$ is performed based on this criterion, therefore the number of clusters is not adjustable and completely related to network properties. In these methods, each node select own $\mathrm{CH}$ based on received messages from its neighbors so the number of clusters is dependent on the transmission range of wireless nodes. If transmission range is increased, the number of clusters is decreased that is suitable in cluster management functions. If the number of nodes in network is too high (or the network is very dense), the number of nodes within a cluster is increased by reducing the number of clusters (or by increasing the transmission range). The management of the cluster with the many number of nodes is difficult. In this paper, we are going to set the input parameter that slightly adjusts the number of clusters when network is very dense. In our algorithm the average number of clusters is suitable in dense network and the average reaffiliation is fewer than some priori methods. 


\section{a. PAMC Algorithm Definition}

We consider a parameter $\mathrm{p}$, where is a real number between 0 and 1 . This parameter is a factor that determines the probability of selecting a node as cluster head. We propose a Probabilistic Algorithm in MANET Clustering (PAMC). In this algorithm, when a node is created, it is marked as $\mathrm{CH}$ with probability of $\mathrm{p}$. This process is described in Algorithm 1. Algorithm 1 is executed only once for each node at the beginning of node creation procedure. After executing Algorithm 1, some "I_am_Head" messages are floating in the network. Each node selects its CH based on "I_am_Head" messages that receive from its neighbors. The average number of clusters increases with the increase parameter $p$, therefore, we can set larger value to $\mathrm{p}$ when the network is very dense.

In Algorithm 2, the $\mathrm{CH}$ determination phase is shown. Algorithm 2 in Step 3 identifies the list of neighbors that send message "I_am_Head". If Heads list is empty, the node marks itself as $\mathrm{CH}$ (Step 4 to 5). Otherwise in Step 7, the member $\mathrm{h} \in$ Heads is selected that has the highest degree and is marked as $\mathrm{CH}$. If two neighbors or more exist with highest degree, the neighbor with lowest ID is selected as CH. In Step 11, if the node is not marked as $\mathrm{CH}$ while has be marked as $\mathrm{CH}$ in before, it should send message "I_am_not_Head" to neighbors. Algorithm 2 is executed after any changing in neighbor list.

\section{b. PAMC Algorithm Analysis}

In this section, we are analyzing our algorithm based on time complexity and message complexity.

Time Complexity: The time complexity of PAMC algorithm is calculated based on summation of time complexity of Algorithm 1 and Algorithm 2. Algorithm 1 is executed with complexity of $\mathrm{O}(1)$. In this algorithm, Step 2, any extra burden is not added to create_node procedure of MANET. Step 3 to 7 that is related to PAMC algorithm is executed in O(1) time. Algorithm 2 is executed in $\mathrm{O}\left(\mathrm{N}_{\mathrm{b}}\right)$ time, where $\mathrm{N}_{\mathrm{b}}$ is the maximum number of neighbors at each node. In this algorithm, all of steps expect Step 7 are executed in O(1) time. In Step 7, the neighbor with the highest degree should be elected; therefore this step is executed in $\mathrm{O}\left(\mathrm{N}_{\mathrm{b}}\right)$ time. Finally, the time complexity of PAMC algorithm is $\mathrm{O}\left(\mathrm{N}_{\mathrm{b}}\right)$.

Message Complexity: In PAMC algorithm, two messages "I_am_Head" and "I_am_not_Head" is sent during network in broadcasting mode. Each node sends these messages to neighbors. If broadcasting algorithm is executed based on spanning tree [3], the message complexity of sending these messages is $O(m)$, where $m$ is the number of edges in the network topology. Therefore, the message complexity of PAMC algorithm is $\mathrm{O}(\mathrm{m})$.

\section{Simulation Environment Results}

In this section, we present the performance of PAMC algorithm by simulation results. We use the Network Simulator 2 (NS2) for our simulation.

\section{a. Simulation Study}

We simulate a network with $\mathrm{N}$ nodes on an $100 \times 100 \mathrm{~m}$ area. $\mathrm{N}$ is varied between 10 and 50. $\mathrm{R}$ is the transmission range that is varied between 0 and 50 meter, and MD is maximum displacement that is varied between 0 and 10 meter. Other simulation parameters are shown in Table 1.

We consider two criteria for performance evaluation of our method. The first criterion is the average re-affiliation that means the average number of changes in $\mathrm{CH}$ at each node during 
network running. The second criterion is the average number of clusters that are created by PAMC algorithm.

\section{Table 1. Simulation Parameters}

\begin{tabular}{|c|c|c|}
\hline Parameter & Meaning & Value \\
\hline $\mathrm{N}$ & Number of nodes & $10-50$ \\
\hline $\mathrm{X} \times \mathrm{Y}$ & Simulation Area & $100 \times 100 \mathrm{~m}$ \\
\hline $\mathrm{R}$ & Transmission Range & $0-50 \mathrm{~m}$ \\
\hline $\mathrm{MD}$ & Maximum Displacement & $0-10 \mathrm{~m}$ \\
\hline Duration & Simulation time & $200 \mathrm{sec}$ \\
\hline $\mathrm{PT}$ & Pause Time & $5 \mathrm{sec}$ \\
\hline Mobility Model & Random way point & -- \\
\hline Simulation & Number of simulation & 10 \\
\hline
\end{tabular}

\section{b. Simulation Results and Discussion}

In this section, we focus to prove the performance of our PAMC in the term of average reaffiliation and the average number of clusters.

The Figure 2(a) shows the variation of the average re-affiliation with respect to the transmission range, where MD is 5. The results are shown for varying N. As shown in Figure 2 , the average re-affiliation decreases with the increase in the transmission range.

The Figure 2(b) shows the variation of the average number of clusters with respect to the transmission range, where MD is 5. The results are shown for varying N. As shown in Figure 2-(b), the average number of clusters decreases with the increase in the transmission range.

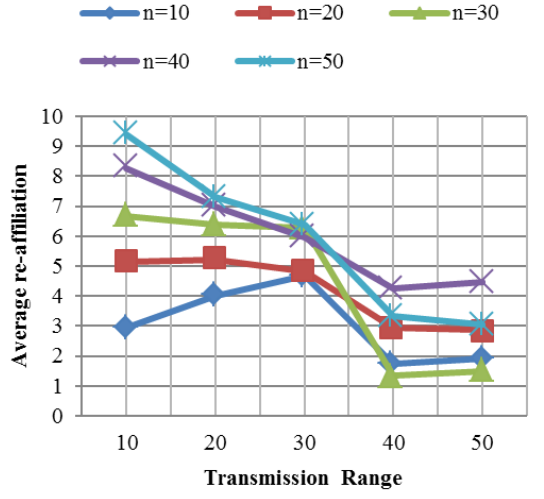

(a)

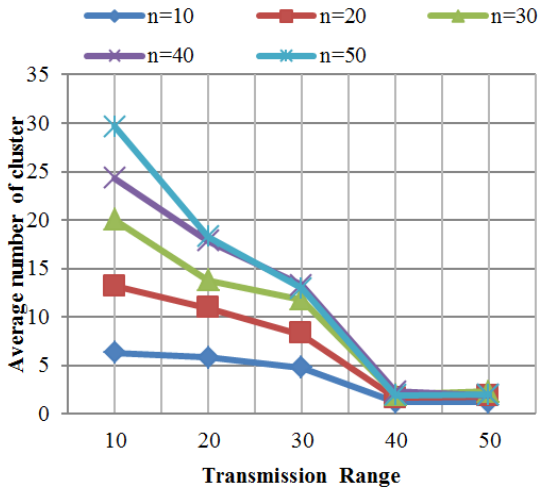

(b)

Figure 2. Relation between Transmission Range and (a) Average Re-affiliation; (b) Average Number of Clusters (MD =5)

Figure 3(a) and 3(b) show the relation between maximum displacement and two criteria, where TR is 30. As shown in Figure 3(a), the average re-affiliation increases with the increase in the maximum displacement. The average number of clusters very slowly increases with the increase in the maximum displacement that this property is good in our algorithm. 


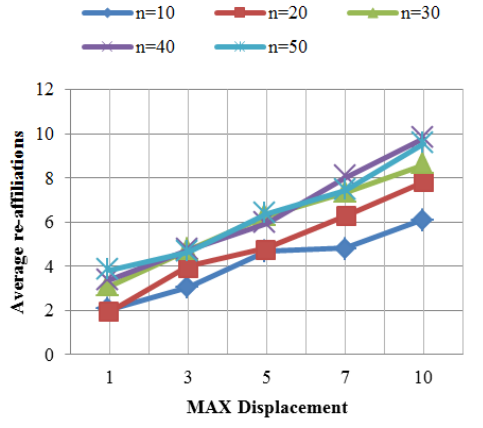

(a)

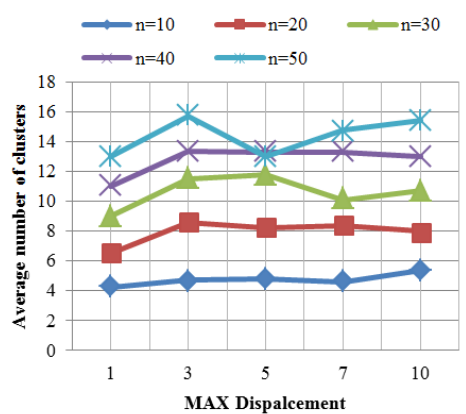

(b)

Figure 3. Relation between Maximum Displacement and (a) Average Reaffiliation; (b) Average Number of Clusters (TR =30)

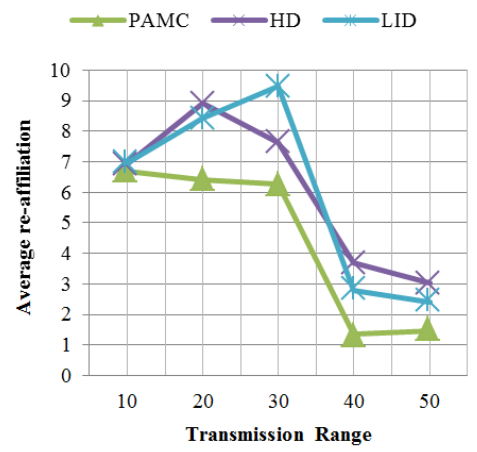

(a)

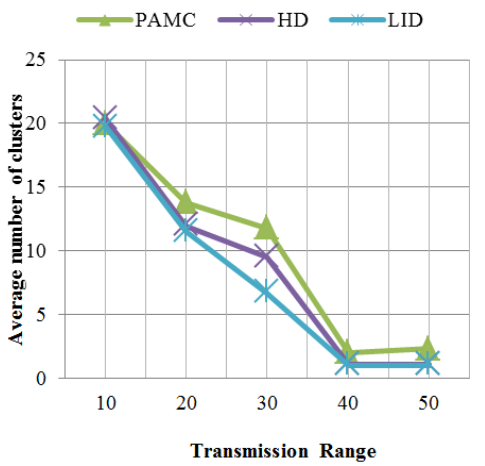

(b)

Figure 4. Relation between the Transmission Range and (a) Average Reaffiliation (b) Average Number of Clusters ( $M D=5, N=30$ )

We also compare PAMC algorithm with LID and HD methods. Figure 4(a) and 4(b) are based on simulation results with 30 nodes and maximum displacement of 5 and show the relation between the transmission range and two criteria. Figure 4(a) proves that the average re-affiliation of PAMC is the lowest value in three methods. Figure 4(b) shows that the average number of clusters is almost 1 in LID and HD methods, if the transmission range is set $50 \mathrm{~m}$, while this property is not good because one cluster head should manage all of nodes that it is inefficient, but in our method at this environment, at least two clusters is created, therefore managing is more efficient.

Figure 5(a) and 5(b) show the variation of the average re-affiliation and the average number of clusters with respect to the transmission range where MD of 5 and $\mathrm{N}$ of 50. Figure 5(a) proves that the average re-affiliation of PAMC method has the best performance of LID and HD methods. Figure 5(b) show that PAMC method creates more clusters than LID and HD methods, if the number of node is very high. This property is good because cluster head should not perform heavy management functions. 


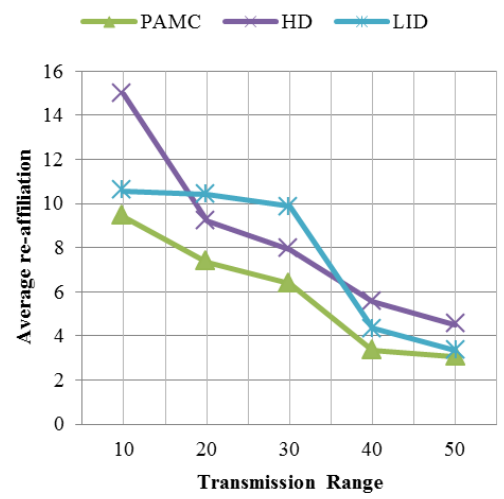

(a)

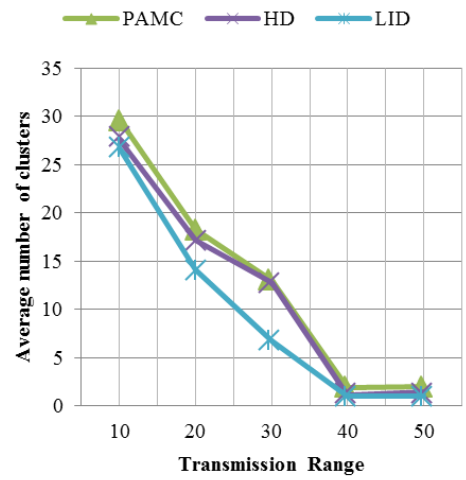

(b)

Figure 5. Relation between the Transmission Range and (a) Average Reaffiliation (b) Average Number of Clusters ( $M D=5, N=50$ )

Figure 6(a) proves that PAMC method has the best average re-affiliation in other methods.

Figure 7(a) and 7(b) show the results in environment that the transmission range and number of nodes are fixed to $30 \mathrm{~m}$ and 50 . These Figures show relation between the maximum displacement and two criteria. Figure 7(a) proves that PAMC algorithm has the best performance in the average re-affiliation criterion

As mentioned, in all of figures based on simulation results, the average re-affiliation of PAMC method is the lowest value that LID and HD methods. This property is very good in MANET because with clustering algorithm, the network should not bear the extra burden.

In PAMC method, the average number of clusters is customizable as possible. Figure 2(a) and 3(a) prove that the average number of clusters is suitable at any size of networks with the transmission range of 30 .

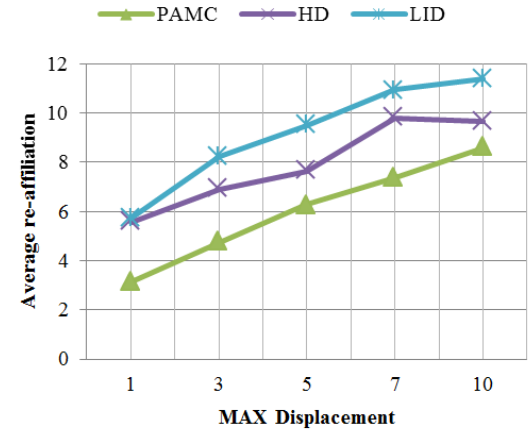

(a)

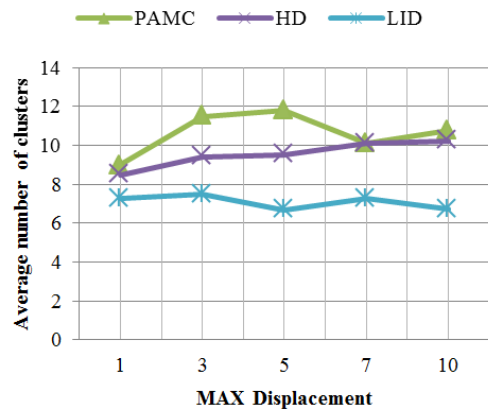

(b)

Figure 6. Relation between the Maximum Displacement and (a) Average Reaffiliation (b) Average Number of Clusters (TR=30, $\mathrm{N}=30$ ) 


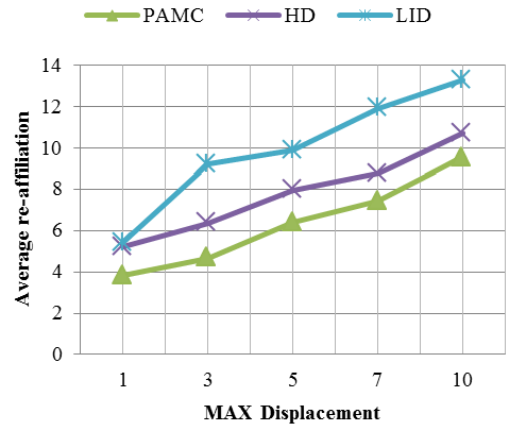

(a)

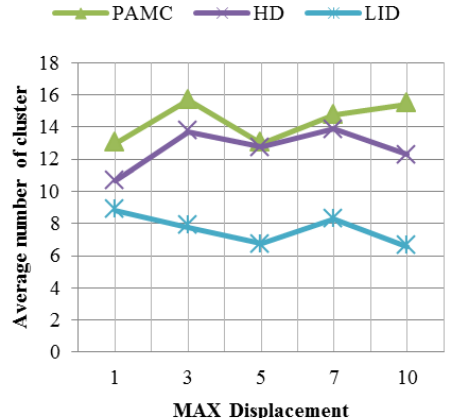

(b)

\section{Figure 7. Relation between the Maximum Displacement and (a) Average re- affiliation (b) Average number of clusters ( $T R=30, N=50$ )}

\section{Conclusion}

In this paper, we propose a probabilistic and heuristic algorithm for clustering nodes in the mobile ad hoc network. Our algorithm has a good performance in the average re-affiliation frequency and the number of clusters is adjusted with probability parameter $\mathrm{p}$. The PAMC algorithm can be used in routing algorithm with low complexity in time $\left(\mathrm{O}\left(\mathrm{N}_{\mathrm{b}}\right)\right)$ and message $(\mathrm{O}(\mathrm{m}))$. With clustering of nodes, we can reduce extra information in routing process. In other words, in the cluster formation, every group of nodes near together arranged to be in one group which is used to prevent the flood of unnecessary packets and avoids wasting network bandwidth.

\section{References}

[1] R. P. Selvam and V. Palanisamy, "An efficient cluster-based multi-source Multicast Routing Protocol in mobile ad hoc networks", IEEE International Conference on Communication Control and Computing Technologies (ICCCCT), (2010), pp. 700 - 706.

[2] Ratish Agarwal and Mahesh Motwani, "Survey of clustering algorithms for manet", International Journal on Computer Science and Engineering, p. abs/0912.2303, (2009).

[3] E. Kayhan, "Distributed graph algorithms for computer networks", Computer Communications and Networks(Springer), (2013).

[4] D. Wei and H. Anthony Chan, "Clustering ad hoc networks: Schemes and classifications", Wirel Netw, vol. 1, (1995), pp. 255-265.

[5] R. S. a. V.Palanisamy, "An efficient clusterbased multi-source multicast routing protocol in mobile ad", IEEE Proc. Communication Control and Computing Technologies (ICCCCT), (2010), pp. 414-419.

[6] D. J. Baker and A. Ephremides, "A distributed algorithm for organizing mobile radio telecommunication networks", in: Proceedings of the 2nd International Conference on Distributed Computer Systems, (1981), pp. 467-483.

[7] D. J. Baker and A. Ephremides, "The architectural organization of a mobile radio network via a distributed algorithm", IEEE Transactions on Communications COM, vol. 29, (1981), pp. 1694-1701.

[8] A. Ephremides, J. E. Wieselthier and D. J. Baker, "A design concept for reliable mobile radio networks with frequency hopping signaling”, in: Proceedings of IEEE, vol. 75, (1987), pp. 56-73.

[9] P. A. K., "Selecting routers in ad-hoc wireless networks", in: Proceedings of the SBT/IEEE International Telecommunications Symposium, (1994).

[10] M. Gerla and J. T.C. Tsai, "Multicluster, mobile, multimedia radio network", Wireless Networks, vol. 1, no. 3, (1995), pp. 255-265.

[11] K. Erciyes, O. Dagdeviren and D. Ozsoyeller, "Distributed algorithms to form cluster based spanning trees in wireless sensor networks”, In: ICCS 2008. LNCS. Springer, (2008), pp. 519-528.

[12] M. Chatterjee, S. K. Das and D. Turgut, "Wca: A weighted clustering algorithm for mobile ad hoc networks", Journal of Cluster Computing (Special Issue on Mobile Ad hoc Networks, vol. 5, (2002), pp. 193-204. 
[13] L. Zou, Q. Zhang and J. Liu, "An improved weight-based clustering algorithm in manets", Wireless Communications, Networking and Mobile Computing, (2008).

[14] M. Shayesteh and N. Karimi, "An innovative clustering algorithm for manet based on cluster stability", International Journal of Modeling and Optimization, vol. 2, (2012), p. 3.

[15] peter h., Jane y. Yu and J. Chong, "A survey of clustering schemes for mobile ad hoc networks", IEEE communications surveys \& tutorials, vol. 7, (2005), p. 1.

[16] A. akbari, S. v. jalali and M. soruri, "Survey of stable clustering for mobile adhoc networks", IEEE Proc., pp. 978-0-7695-3944-7/10, (2010). 
International Journal of Future Generation Communication and Networking Vol. 7, No. 6 (2014) 